\title{
Expression of Biomarkers in Canine Mammary Tumours
}

\author{
N. Muniappan*, S. Ramesh, S. Hemalatha, Mohamed Shafiuzama and S.P. Preetha
}

Department of Veterinary Pharmacology and Toxicology, Madras Veterinary College, Chennai-7, Tamil Nadu Veterinary and Animal Sciences University, India

*Corresponding author

\begin{tabular}{|c|}
\hline Keywords \\
\hline $\begin{array}{l}\text { Canine mammary } \\
\text { tumours, } \\
\text { Biomarkers } \\
\text { immnuno } \\
\text { histochemistry }\end{array}$ \\
\hline Article Info \\
\hline $\begin{array}{l}\text { Accepted: } \\
12 \text { April } 2019 \\
\text { Available Online: } \\
10 \text { May } 2019\end{array}$ \\
\hline
\end{tabular}

\section{Introduction}

The incidence of tumours in canine is on the rise in the past decade and mammary tumors

are the second most common canine tumours

\section{A B S T R A C T}

Biomarkers identifiable in tumours have potential use in early diagnosis, staging, prognosis and assessment of response to therapy. This paper describes the immunohistochemical expression of a few reliable biomarkers in post surgical canine mammary gland tumour (CMGT). Immunohistochemical expression of biomarkers in canine mammary tumours was carried out in forty three mammectomised tissue samples. The intensity and distribution of immunostaining of the biomarkers in histological types of CMGT was studied. PCNA showed weak nuclear staining in benign tumour and strong nuclear staining in malignant tumours. Bcl 2 showed weak cytoplasmic staining in benign tumours and moderate cytoplasmic staining in 10-20\% of epithelial cells in malignant tumours. Cytokeratin $8 / 18$ expression was strong in $>50 \%$ of epithelial cells in benign and malignant tumours. Vimentin expression was specific with strong cytoplasmic staining in the stromal fibrous tissue, stromal fusiform cells, myoepithelial cells in tubules, spindle cells, chondroid tissue and lipocytes. CEA expression was negative in benign tumours. Malignant tumours expressed moderate membranous to cytoplasmic staining in $10-20 \%$ of luminal epithelial cells. MUC 1 expression was weak membranous staining in $<10 \%$ of apical portion of luminal epithelial cells in benign tumours. Overexpression of MUC 1 was observed as moderate membranous and cytoplasmic staining in 10-20\% of luminal epithelial cells in malignant tumours. HER-2 expression was weak membrane staining in $<10 \%$ of luminal epithelial cells in benign tumours. Moderate membranous and cytoplasmic staining was observed in $10-20 \%$ of luminal epithelial cells in malignant tumours. COX 2 enzyme expression was negative in benign tumours. Moderate cytoplasmic staining in $10-20 \%$ of luminal epithelial cells, myoepithelial cells, stromal fusiform cells and stromal inflammatory cells was observed in malignancy. Hence the expression of biomarkers in tissues is of diagnostic and prognostic significance before initiation of treatment regimen in canine mammary tumours. with increased frequency among female dogs. About $50 \%$ of the canine mammary gland tumours are diagnosed as malignant. Canine mammary gland tumours (CMGT) are generally classified based on their histological 
appearances and the grade of malignancy is based not only on tumor type but also on the presence of significant nuclear and cellular pleomorphism, mitotic index, the presence of necrotic areas, peritumoral, lymphatic invasion and regional lymph node metastasis (Goldschmidt et al., 2011). Potential applications of biomarkers in CMGT help in early diagnosis, staging, prognosis and monitoring responses to therapy. Tumour specific biomarkers are used to monitor remission status and initiation of therapy and improve clinical outcome (Henry, 2010). Various biomarkers are used in CMGT due to its heterogenous morphology and biological behaviour. This paper describes the immunohistochemical expression of a few reliable biomarkers in post surgical CMGT carried out preliminarily for diagnostic and prognostic purpose and for initiation of an experimental chemotherapeutic regimen for CMGT.

\section{Materials and Methods}

The mammary tumour samples consisted of surgically excised specimens from clinical cases of dogs referred to Small Animal Clinic, Out Patient Surgery Unit, Madras Veterinary College Teaching Hospital, Chennai, Tamil Nadu. The study was carried out during the period from April 2016 to April 2019. Forty three mammectomised tissue samples were fixed in $10 \%$ neutral buffered formalin immediately after surgery, routine histological processing was carried out, tissues were embedded in paraffin wax and sections of 4-5 $\mu \mathrm{m}$ were stained with hematoxylin and eosin for histopathological examination (Bancroft and Stevens, 1996). Classification and grading of lesions on canine mammary tissues were carried out according to the diagnostic criteria proposed by Goldschmidt et al., (2011). Histological studies yielded 7 benign tumours (19.44\%) and 36 malignant tumours $(83.72 \%)$ among the total number of 43 canine mammary gland tumours recorded during the period of study. Immunohistochemistry was carried out in representative tissue samples from each histological tumour type.

Immunostaining for proliferation cell nuclear antigen (PCNA), carcinoembroyonic antigen (CEA), cancer antigen (CA15-3), epidermal growth factor HER2, cyclooxygenase enzyme $\mathrm{COX} 2$, vimentin, apoptotic marker $\mathrm{Bcl} 2$ was carried out using 3-4 $\mu \mathrm{m}$ thick paraffin sections collected on 3-aminopropyl methoxy-silane coated microslides and dried at $60^{\circ} \mathrm{C}$ for $1 \mathrm{~h}$. Sections were deparaffinised in xylene for $3 \mathrm{~min}$, xylene and alcohol (1:1) for $3 \mathrm{~min}$ and then washed in graded of alcohols. After washing in running tap water the slides were placed in antigen retrieval buffer (PathnSitu Cat PS007) for $30 \mathrm{~min}$ under steam pressure for antigen retrieval. The sections were incubated with peroxide block and protein block $5 \mathrm{~min}$ each and treated with primary rabbit monoclonal antibodies (PCNA- EP91 (PathnSitu Cat No.PR065); CK8:EP17; CK18:EP30 (PathnSitu Cat No.PR 0321); CEA COL-1 (PathnSitu Cat No.PM086); Bcl-2 EP36 (PathnSitu Cat No.PR004); COX2-SP21 (PathnSitu Cat No.PR180); HER2-EP3 (PathnSitu Cat No.PR047); MUC1-EP85 (PathnSitu Cat No.PR149); Vimentin EP21 (PathnSitu Cat No PR075), for $30 \mathrm{~min}$ in a moist chamber at room temperature. The primary antibodies was omitted and replaced with PBS for negative control. Washing after every incubation was carried out using immunewash buffer (PathnSitu Cat PS006). The sections were incubated in PolyExcel HRP labeled polymer for $30 \mathrm{~min}$ at room temperature and staining was completed with 3,3'-diaminobenzidine (DAB) substratechromogen for $5 \mathrm{~min}$ at room temperature (PathnSitu Cat No.OSH001) and counter stained with haematoxylin. Colon tissue was used as positive control for MUC1, 
cytokeratin, Cox2 and CEA; human breast cancer tissue was used as positive control for HER2 expression. Positive immunostaining was assessed by the presence of golden brown granular labeling in the cell membrane, cytoplasm or nucleus of the CMGT cells based on the biomarker studied. The distribution of positive immunostained cells was graded as 0-nostaining; 1-1-10\%; 2-11$20 \% ; 3-51-80 \% ; 4-81-100 \%$ and the intensity of staining was recorded as negative -0 ; weakly positive + ; moderate positive $2+$; strong positive $3+$.

\section{Results and Discussion}

The intensity and distribution of immunostaining of the biomarkers studied in histological types of CMGT are given in Table 1.

PCNA is a marker of the proliferation index. PCNA is an auxiliary protein of DNA polymerase $\delta$, which is expressed in the nuclei of cells during the DNA synthesis phase of the cell division cycle. It is also involved in the DNA repair process, cell cycle control, chromatin assembly and in RNA transcription (Jurikovaa et al., 2016). PCNA level was frequently evaluated in cases of mammary cancer, its expression was greater in tumours that showed more malignancy, large tumor size, skin ulceration, histological grade II or III, and presence of regional lymph node metastases (Carvalho et al., 2016).

Weak expression of PCNA was seen in nucleus in $<10 \%$ of luminal epithelial cells in benign CMGT. Strong nuclear staining in $>20 \%$ of luminal epithelial cells was observed in tubular carcinomas (Fig. 1) and its histological subtypes as tubulopapillary, cystic papillary, micropapillary and variants like solid, mixed, lipid rich, mucinous, spindle cell and complex carcinomas. Squamous cell carcinoma and fibrosarcoma showed strong nuclear staining in 10-20\% of keratinised epithelial cells and stromal fusiform cells. PCNA expression in the present study was similar to the report of Zucarri et al., (2008).

Apoptosis is regulated by a wide variety of factors, especially the Bcl-2 family: inhibitors i.e. Bcl-2 protein. Dolka et al., (2016) demonstrated that most of the Bcl-2-positive tumours were benign. In addition, Bcl-2 was strongly expressed in benign lesions, in the grade I and II differentiated carcinomas, and in dogs with lower clinical stages of tumour.

Weak cytoplasmic staining of Bcl-2 expression was seen in $<10 \%$ of luminal epithelial cells in benign adenomas. Tubular carcinoma, its histological subtypes and variants showed moderate cytoplasmic staining in 10-20\% of luminal epithelial cells (Fig. 2). Squamous cell carcinoma showed moderate cytoplasmic staining in $10-20 \%$ of keratinised epithelial cells. Fibrosarcoma showed weak cytoplasmic staining in $<10 \%$ of fusiform cells. The findings in our study was also in accordance to the above reference for malignant tumours, however benign tumours showed weakly positive immunostaining.

Intermediate filaments (IFs) are one of the most important types of tumour markers, whose presence or absence is very important. A large group of IFs known as cytokeratins, which are specific for epithelial cells and carcinomas. Most malignant tumours are highly consistent, due to their strong cytoskeleton which is made of cytokeratin. In our study, strong cytokeratin expression in cytoplasm was seen in $>50 \%$ of luminal epithelial cells in benign and malignant tubular carcinomas, its histological subtypes and variants (Fig. 3). Fibroadenoma and mixed carcinoma showed moderate cytoplasmic staining in $10-20 \%$ of luminal 
epithelial cells. Squamous cell carcinoma showed strong cytoplasmic staining in $>50 \%$ of keratinsed epithelial cells. Fibrosarcoma showed negative staining. The findings in this study was in accordance with the reports of Eivani and Mortazavi (2016) and Sorenmo et al., (2011) which stated that the luminal epithelial cells are characterized by the expression of low molecular weight luminal cytokeratins (CKs), including CK8, CK18.

Vimentin is a member of class-III intermediate filaments and it is especially found in mesenchymal cells, because of this, it is used as a mesenchymal and myoepithelial cell marker in the tumours. The overexpression of vimentin filaments in types of cancer of an epithelial origin is abnormal and represents phenotypic changes of malignant epithelial cells into mesenchymal cells, known as epithelial-mesenchymal transition (EMT). The evaluation of vimentin expression in mammary tumour is considered to be beneficial for determining the prognosis and has therapeutic value as the tumor progression may be controlled if vimentin and its upstream line are targeted (Satelli and Li,2011). In our study, vimentin expression was weak to moderate cytoplasmic staining in $<10 \%$ or $10-20 \%$ of stromal tissue and stromal fusiform cells in benign ductal and simple adenomas. Chondroid tissue in mixed adenoma showed positive staining. Fibroadenoma showed strong cytoplasmic staining in stroma. Tubular carcinoma, its histological subtypes showed strong cytoplasmic staining in $10-20 \%$ of stromal tissue and stromal fusiform cells (Fig. 4) and myoepithelial cells (Fig. 5). Solid carcinoma showed weak cytoplasmic staining in the minimal stromal tissue and stromal fusiform cells. Mixed carcinoma showed strong cytoplasmic staining in $10-20 \%$ of dense fibrous tissue stroma, fusiform cells, myoepithelial cells in tubules and in chondroid matrix. Squamous cell carcinoma showed weak staining in minimal stromal tissue whereas strong cytoplasmic staining was observed in $>80 \%$ of fibrous tissue and anaplastic or fusiform cells in fibrosarcoma. Lipid rich carcinoma and mucinous carcinoma showed moderate cytoplasmic staining in $<10 \%$ of lipocytes, stromal tissue and fusiform cells. Spindle cell carcinoma and complex carcinoma showed moderate to strong cytoplasmic staining in 10-20\% of stromal tissue, fusiform cells in stroma and spindle cells or myoepithelial cells in carcinoma. The results of our study corroborated with that of Aydogan and Metin (2013) in a study to identify the cell of origin in CMGT wherein mesenchymal and myoepithelial cells expressed positive for vimentin in all mammary tumour cases studied.

CEA is a glycoprotein that is involved in intracellular adhesion. It is a glycoprotein produced by gastrointestinal mucosa, localized in epithelial cell membranes in small amounts, and it is overexpressed by the cancer cells of the colon, breast and lungs. It is usually measured in serum using various immunochemical methods, such as radioimmunological methods (RIA) or electrochemistry luminescence immunoassay (ECL) (Shao et a1.2015). It is also a useful marker for early detection of recurrence and metastasis.

CEA expression was negative in benign tumours. Tubular carcinoma, its histological subtypes and variants like lipid rich, mucinous, spindle cell and complex carcinomas showed moderate membranous to cytoplasmic staining in $10-20 \%$ of luminal epithelial cells (Fig. 6). Overexpression of CEA was observed in solid carcinoma and weak cytoplasmic staining in $<10 \%$ of luminal epithelial cells in mixed carcinoma, keratin epithelial cells in squamous cell carcinoma and anaplastic or fusiform cells in 
fibrosarcoma. However, CEA expression in CMGT tissues by immunostaining is less documented and hence the results in our study could not be compared.

CA 15-3 (also called Mucin 1 or MUC 1) is a large transmembrane glycoprotein, a product of the mucinlgene, expressed in the apical plasma membrane. However, during malignant transformation, it can be overexpressed on the membrane surface, as well as in the cytoplasm. During the malignancy process, MUC1 often acts as an anti-adhesive molecule and enables the detachment of malignant cells; therefore, it increases the metastatic and invasive potential of tumour cells (Manuali et al., 2012). Apical cellular localisation in tumour cells is considered to be an indicator of an intact MUC1 pathway and is associated with functional differentiation and a good prognosis. The presence of an aberrant pattern of MUC1 expression, such as cytoplasmic staining, was observed in canine malignant mammary tumours indicative of poor functional differentiation and associated with worse prognosis (Rahn et al., 2001).

Weak staining of MUC 1 in membrane in $<10 \%$ of apical portion of luminal epithelial cells in benign tumours. Overexpression of MUC 1 was observed as moderate membranous and cytoplasmic staining in 10$20 \%$ of luminal epithelial cells in tubular carcinoma (Fig. 7) and its histological subtypes. Strong membranous and cytoplasmic staining was observed in 10-20\% of anaplastic cells in solid carcinoma. Mixed carcinoma, lipid rich, mucinous and spindle cell carcinoma showed weak membranous and cytoplasmic staining in $<10 \%$ of luminal epithelial and spindle cells. Squamous cell carcinoma and fibrosarcoma showed negative staining. Our results were in agreement with earlier studies on overexpression of MUC 1 as membranous and cytoplasmic staining in canine malignant mammary tumours (Oliveira et al., 2009).

HER-2 is also considered an important tumour marker. HER-2 functions to regulate tumour growth, survival and differentiation. It is expressed in approximately $30 \%$ of CMGT, which is the same percentage as in human breast carcinoma (Campos et al., 2015). There is also a positive correlation between HER-2 expression and tumour mitotic index, high histological grade and size (Dutra et al., 2004). HER2 overexpression is associated with malignancy, high histological tumor grade, large tumor size, and p53 expression, suggesting a role in canine mammary gland carcinogenesis and a poor prognosis (Bertagnolli et al., 2011). HER-2 may participate in tumour formation, but not necessarily in malignant transformation, or at least, it is not a good marker of malignancy (Kaszak et al., 2018).

Weak HER-2 expression was seen in $<10 \%$ of luminal epithelial cells in benign tumours. Moderate membranous and cytoplasmic staining was observed in $10-20 \%$ of luminal epithelial cells in tubular carcinoma (Fig. 8) and its histological subtypes. Strong membranous and cytoplasmic staining was observed in 10-20\% of anaplastic cells in solid carcinoma. Variants of carcinoma like mixed, lipid rich, mucinous, spindle cell and complex carcinomas showed moderate cytoplasmic staining in $10-20 \%$ of luminal epithelial cells and spindle cells. Squamous cell carcinoma and fibrosarcoma showed negative staining. Overexpression of HER2 as membranous and cytoplasmic staining in malignant higher grade CMGT in our study was similar to earlier reports as discussed above.

The cyclooxygenase enzyme catalyzes the prostaglandin biosynthesis from arachidonic acid. Many studies have confirmed that 
prostaglandins play an important role in tumor development. Deregulation of the enzymatic pathway of prostaglandin E2 formation is strongly related to neoplasm progression. COX-2 is undetectable in normal tissues; it is expressed in tissue due to inflammatory reactions, growth factors, tumor promoters and oncogenes. Many studies have proven the presence of COX-2 expression in mammary tumors, as well as in some normal mammary tissues (Queiroga et al., 2010).

Table.1 Histological expression of biomarkers in canine mammary gland tumours

\begin{tabular}{|c|c|c|c|c|c|c|c|c|c|c|c|c|c|c|c|c|}
\hline \multirow[t]{2}{*}{ Tumour type } & \multicolumn{2}{|c|}{ PCNA } & \multicolumn{2}{|c|}{ Bcl-2 } & \multicolumn{2}{|c|}{ CK } & \multicolumn{2}{|c|}{ Vimentin } & \multicolumn{2}{|c|}{ CEA } & \multicolumn{2}{|c|}{ MUC1 } & \multicolumn{2}{|c|}{ HER2 } & \multicolumn{2}{|c|}{ COX2 } \\
\hline & $\mathrm{I}$ & G & I & G & I & G & I & G & I & G & I & G & I & G & I & G \\
\hline Ductal adenoma & + & 1 & + & 1 & $3+$ & 3 & + & 2 & - & 0 & + & 1 & + & 1 & - & - \\
\hline Mixed adenoma & + & 1 & + & 1 & $3+$ & 2 & $2+$ & 2 & - & 0 & + & 1 & + & 1 & - & - \\
\hline Simple adenoma & + & 1 & + & 1 & $3+$ & 3 & + & 1 & - & 0 & + & 1 & + & 1 & - & - \\
\hline Fibroadenoma & + & 1 & - & 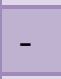 & $2+$ & 2 & $3+$ & 3 & - & 0 & + & 1 & + & 1 & - & - \\
\hline Tubular carcinoma & $3+$ & 3 & $2+$ & 2 & $3+$ & 3 & $3+$ & 2 & $2+$ & 2 & $2+$ & 2 & $2+$ & 2 & $2+$ & 2 \\
\hline $\begin{array}{l}\text { Tubulo papillary } \\
\text { carcinoma }\end{array}$ & $3+$ & 3 & $2+$ & 2 & $3+$ & 3 & $3+$ & 2 & $2+$ & 2 & $2+$ & 2 & $2+$ & 2 & $2+$ & 2 \\
\hline $\begin{array}{l}\text { Cystic papillary } \\
\text { tubular carcinoma }\end{array}$ & $3+$ & 3 & 0 & 2 & + & 3 & + & 2 & $2+$ & 2 & $2+$ & 2 & $2+$ & 2 & $2+$ & 2 \\
\hline $\begin{array}{l}\text { Micropapillary } \\
\text { carcinoma }\end{array}$ & $3+$ & 3 & $2+$ & 2 & $3+$ & 3 & $3+$ & 2 & $2+$ & 2 & + & 2 & $2+$ & 2 & $2+$ & 2 \\
\hline Solid carcinoma & $3+$ & 3 & $2+$ & 2 & + & 2 & + & 1 & $3+$ & 2 & $3+$ & 2 & $3+$ & 2 & $2+$ & 2 \\
\hline Mixed carcinoma & $3+$ & 3 & $2+$ & 1 & $2+$ & 3 & $3+$ & 2 & + & 1 & + & 1 & + & 2 & + & 1 \\
\hline $\begin{array}{l}\text { Squamous cell } \\
\text { carcinoma }\end{array}$ & $3+$ & 3 & $2+$ & 2 & $3+$ & 3 & + & 1 & + & 1 & - & - & - & - & $2+$ & 2 \\
\hline Fibrosarcoma & $3+$ & 3 & + & 1 & - & - & $3+$ & 4 & + & 1 & - & - & - & - & - & - \\
\hline $\begin{array}{l}\text { Lipid rich tubular } \\
\text { carcinoma }\end{array}$ & $3+$ & 2 & $2+$ & 1 & $3+$ & 3 & $2+$ & 1 & $2+$ & 2 & + & 1 & $2+$ & 2 & - & - \\
\hline $\begin{array}{l}\text { Mucinous tubular } \\
\text { carcinoma }\end{array}$ & $3+$ & 3 & $2+$ & 1 & $3+$ & 3 & $2+$ & 1 & $2+$ & 2 & $2+$ & 2 & $2+$ & 2 & + & 1 \\
\hline $\begin{array}{l}\text { Spindle cell } \\
\text { carcinoma }\end{array}$ & $3+$ & 3 & $2+$ & 1 & $3+$ & 3 & $3+$ & 3 & $2+$ & 2 & + & 1 & $2+$ & 2 & $2+$ & 2 \\
\hline $\begin{array}{l}\text { Complex tubular } \\
\text { carcinoma }\end{array}$ & $3+$ & 3 & $2+$ & 1 & $3+$ & 3 & $2+$ & 2 & $2+$ & 2 & + & 1 & $2+$ & 2 & $2+$ & 2 \\
\hline
\end{tabular}

I -Staining intensity + weak; $2+$ moderate; $3+$-strong

$\mathrm{G}-1-10 \% ; 2-11-20 \% ; 3-51-80 \% ; 4-81-100 \%$ 


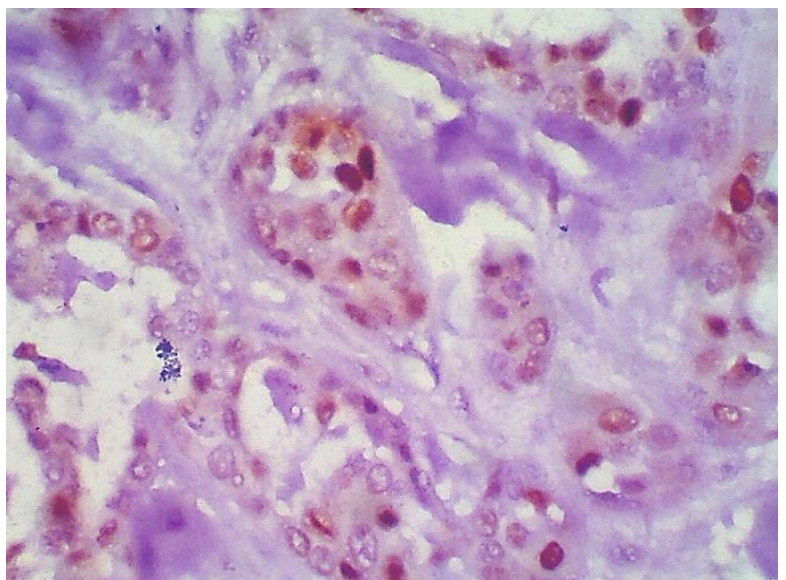

Fig.1 PCNA-Tubular carcinoma- Moderate nuclear immunosatining of epithelial cells DAB/Haematoxylin 400x

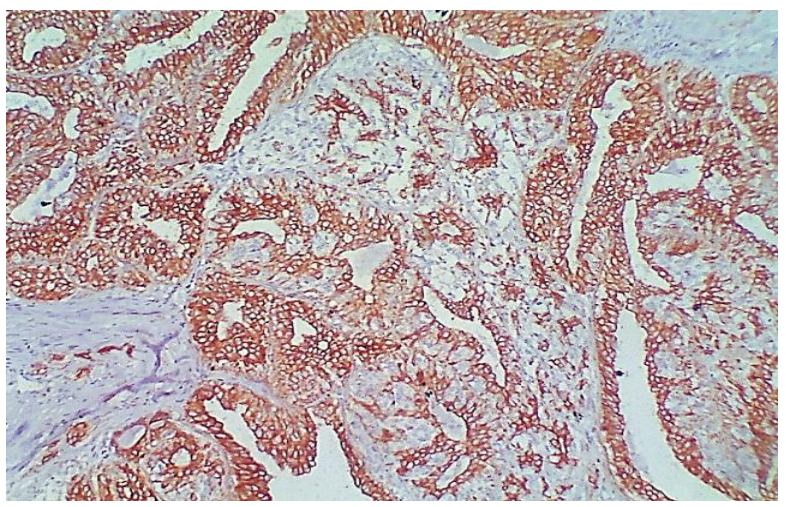

Fig.3 Cytokeratin - Tubulopapillary carcinoma - Strong cytoplasmic immunostaining of luminal epithelial cells DAB/Haematoxylin 100x

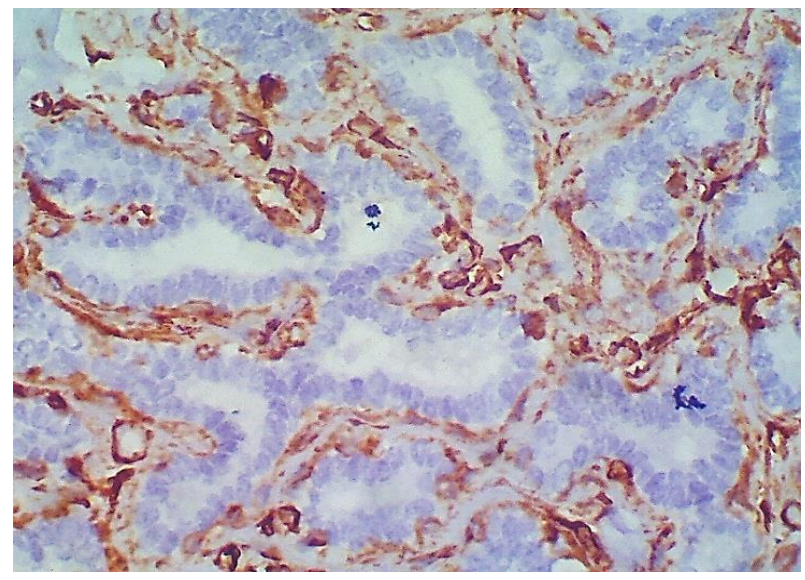

Fig.5 Vimentin-Tubular carcinoma- Strong cytoplasmic immunostaining of myoepithelial cells in tubules DAB/Haematoxylin 400x

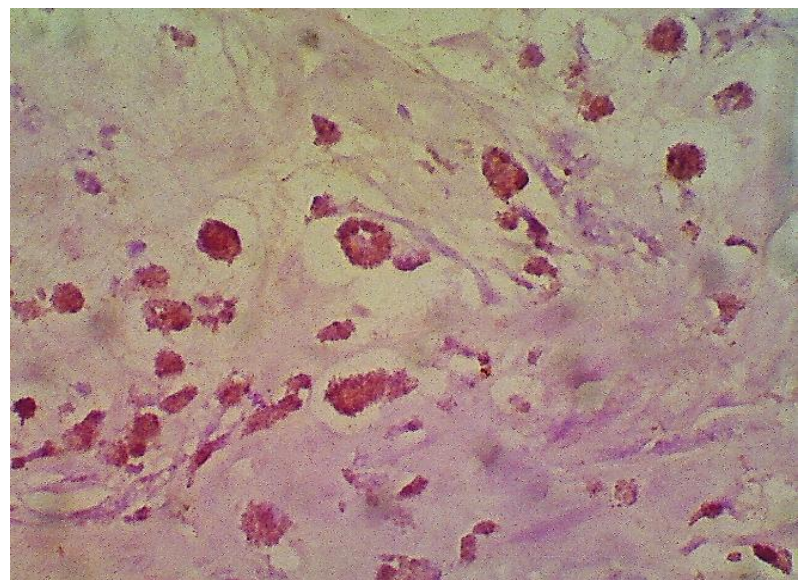

Fig.2 Bcl-2 -Tubular carcinoma- Moderate cytoplasmic immunostaining of epithelial cells DAB/Haematoxylin 400x

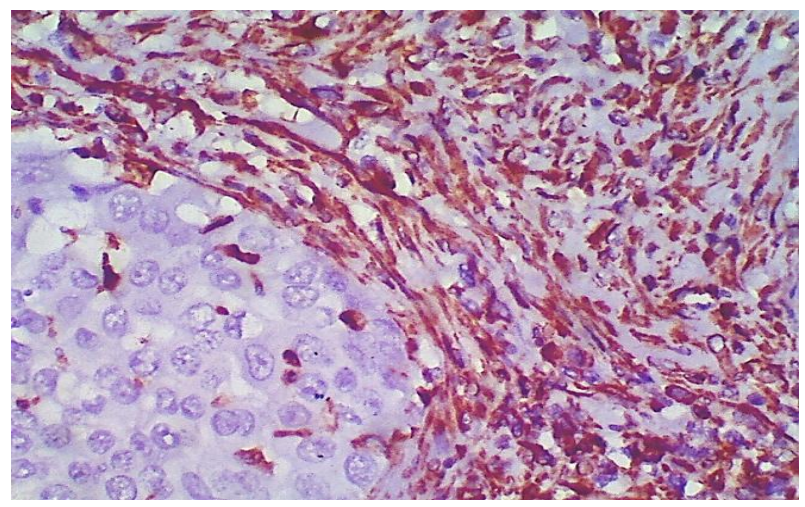

Fig.4 Vimentin-Tubular carcinoma- Strong cytoplasmic immunostaining of stromal tissue and stromal fusiform cells DAB/Haematoxylin 400x

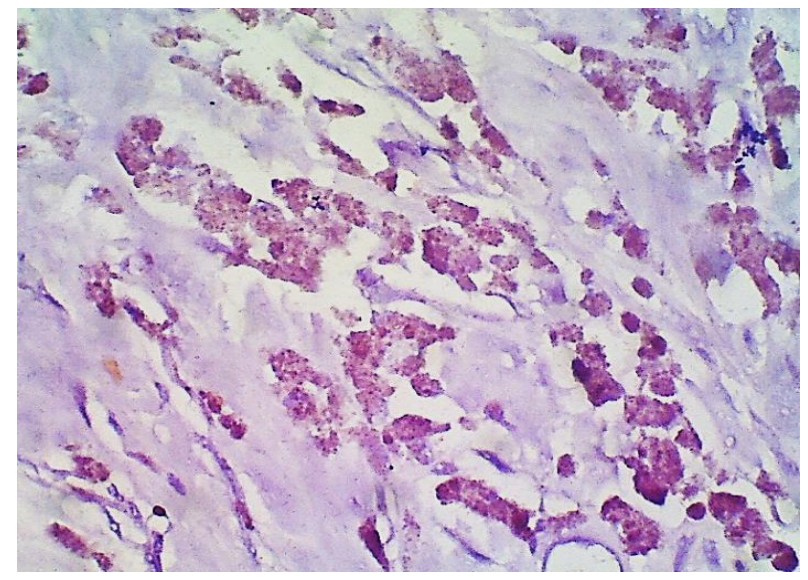

Fig.6 CEA-Tubular carcinoma- Moderate cytoplasmic immunostaining of invasive epithelial cells DAB/Haematoxylin 400x 


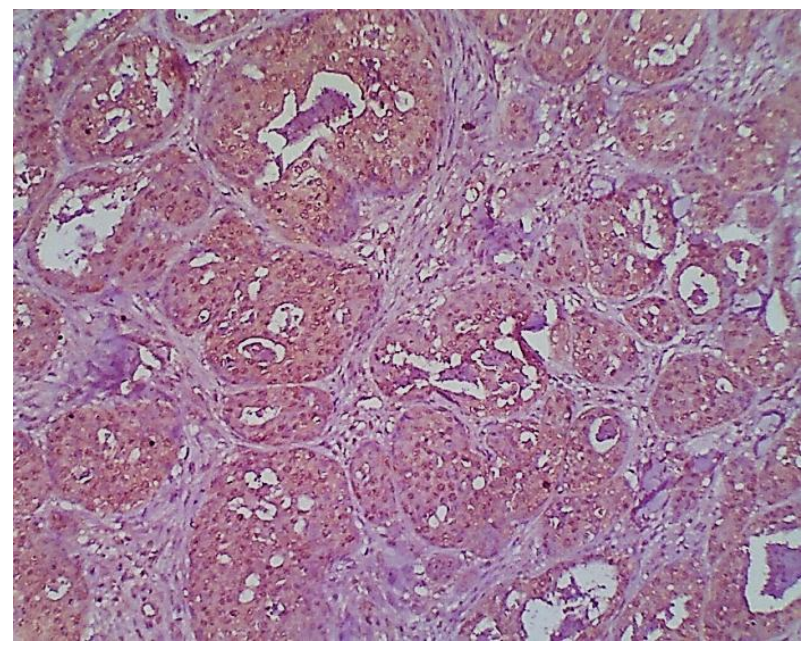

Fig.7 MUC 1-Tubular carcinoma- Moderate cytoplasmic immunostaining of epithelial cells DAB/Haematoxylin 100x

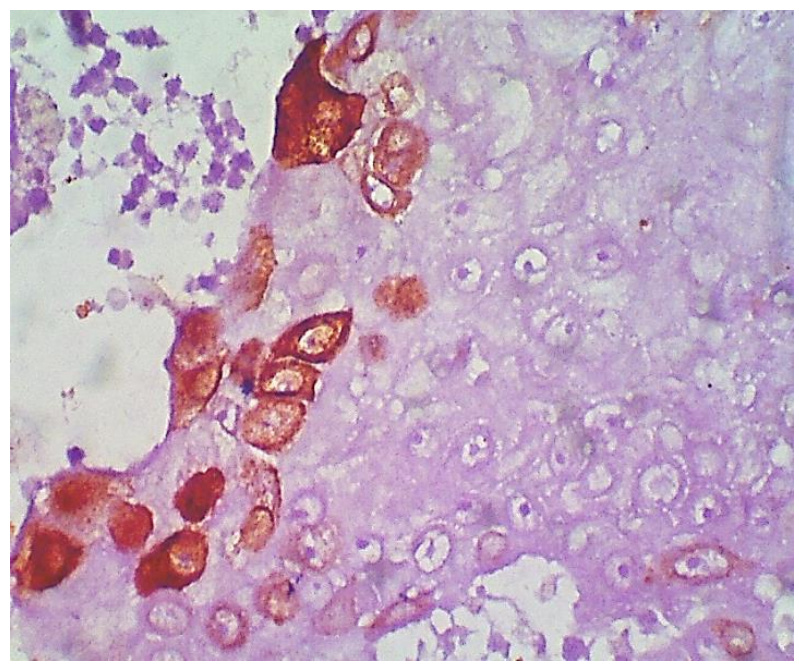

Fig.9 COX-2 -Solid carcinoma- Strong cytoplasmic immunostaining of epithelial cells DAB/Haematoxylin 400x

COX-2 enzyme expression was negative in benign tumours. Moderate cytoplasmic staining in $10-20 \%$ of luminal epithelial cells, myoepithelial cells, stromal fusiform cells and stromal inflammatory cells in tubular carcinoma and its histological subtypes and variants like solid carcinoma (Fig. 9 and 10), spindle cells and complex tubular carcinoma was observed. COX- 2 enzyme was weakly expressed in $<10 \%$ of luminal epithelial cells in mixed and mucinous carcinoma. The

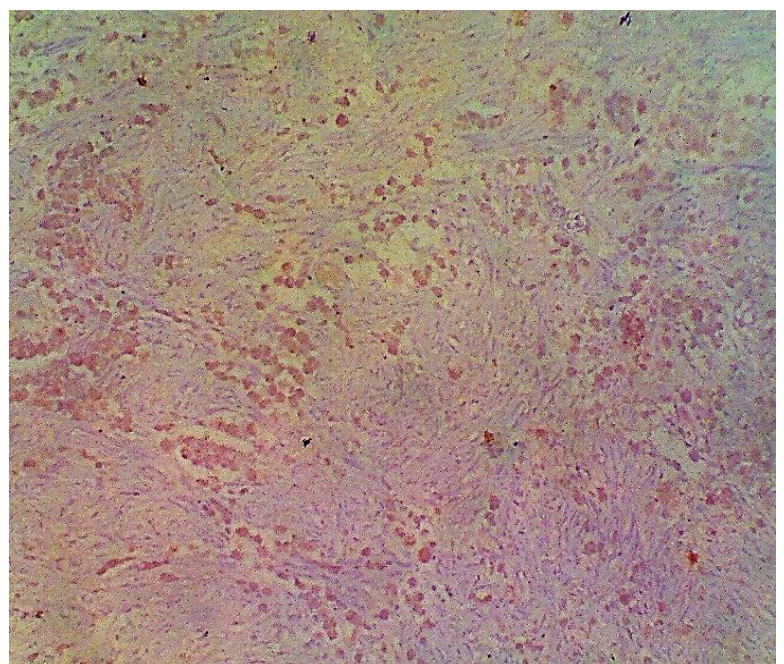

Fig.8 HER2-Tubular carcinoma- Moderate membranous and cytoplasmic immunostaining of epithelial cells DAB/Haematoxylin 100x

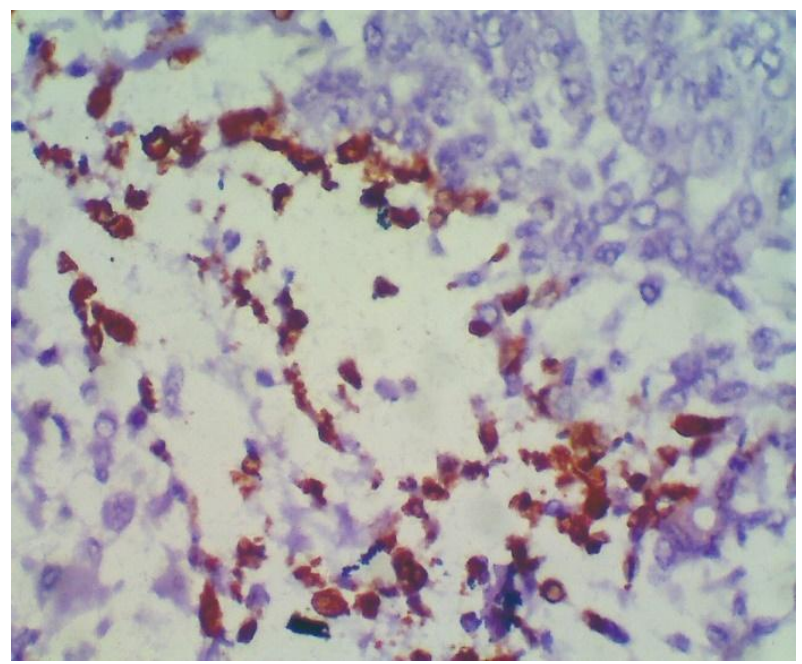

Fig.10 COX-2 -Solid carcinoma- Strong cytoplasmic immunostaining of inflammatory cells DAB/Haematoxylin 400x

keratinised epithelial cells and inflammatory cells on squamous cell carcinoma showed moderate cytoplasmic staining in $10-20 \%$ of cells. Fibrosarcoma showed negative staining. Expression of COX-2 in our study corroborated with the report of Dore et al., (2003) who demonstrated for the first time that a proportion of canine mammary tumors expressed significant amount of COX-2, suggesting the potential value of anti-COX-2 therapies for the treatment of this subset of 
canine mammary tumors. Of the malignant tumours, carcinosarcomas and tubulopapillary and squamous cell carcinomas had the highest COX-2 scores. The study showed that malignant tumours had the highest values of COX-2 expression, and COX-2 immunolabelling was particularly intense in histological types classically associated with high malignancy. This suggested that nonsteroidal anti-inflammatory drugs (NSAIDs), particularly COX-2 inhibitors, might have a useful role to play in the treatment of canine malignant mammary tumours (Queiroga et al., 2010). COX 2 expression in our study also concurred with the findings of the above mentioned workers in CMGT.

Hence the expression of a panel of tissue biomarkers in post surgical CMGT biopsies may be of diagnostic and prognostic significance and also for assessment of response to chemotherapy.

\section{References}

Aydogan, A., and Metin, N. 2013. Detection of cell origin by immunohistochemistry in canine mammary tumours. Revue de Médecine Vétérinaire. 164(7): 395-399.

Bancroft, J., Stevens, D.A. and Turner, D.R. 1996. Theory and Practice of Histological Techniques, III Edn. Churchill Livingstone, London.

Bertagnolli, A.C., 2011. Canine mammary mixed tumours: immunohistochemical expressions of EGFR and HER-2. The Australian Veterinary Journal. 89:312317.

Campos, L.C., Silva, J.O, Santos, F.S., Araujo, M.R, Lavalle, G.E and Ferreira, E. 2015. Prognostic significance of tissue and serum HER2 and MUC1 in canine mammary cancer. Journal of veterinary diagnostic investigation. 27:531-535.
Carvalho, M.I., Pires, I and Prada, J. 2016. Ki-67 and PCNA expression in canine mammary tumors and adjacent nonneoplastic mammary glands: prognostic impact by a multivariate survival analysis. Veterinary Pathology. 53:1138-1146.

Dolka, I., Król, M, Sapierzyński, R. 2016. Evaluation of apoptosis-associated protein (Bcl-2, Bax, cleaved caspase3and p53) expression in canine mammary tumors: An immunohistochemical and prognostic study. Research in Veterinary Science 105: 124-133

Dore, M., Lanthier, I and Sirois, J. 2003. Cyclooxygenase-2 Expression in Canine Mammary Tumors. Veterinary Pathology.40:207-212.

Dutra, A.P., Granja, N.V. and Schmitt, F.C. 2004. c-erbB-2 expression and nuclear pleomorphism in canine mammary tumors. Brazilian Journal of Medical and Biological Research. 37:1673-168.

Eivani, D., and Mortazavi, D. 2016 The relationship between basal and luminal cytokeratins with histopathologic characteristics of canine mammary gland cancer. Polish Journal of Veterinary Sciences. 19(2): 2261-269.

Goldschmidt, M.1., Peña, L, Rasotto, R and Zappulli, V. 2011. Classification and grading of canine mammary tumors. Veterinary Patholology. 48(1):117-3.

Henry, J.C., 2010. Biomarkers in veterinary cancer screening: applications, limitations and expectations. The Veterinary Journal. 185:10-14.

Kaszak, I., Ruszczak, A, Kanafa, S, Kacprzak K, Król, M. and Jurka, P. 2018. Current biomarkers of canine mammary tumors. Acta Veternaria Scandinavia. 60:66

Oliveira T de. A, Salomé. S, Pinho. A, Augusto. J, De Matos B, Venceslau Hespanhol. C, Celso. A, Reis. A.C and Fátima Gärtner. 2009. MUC1 
expression in canine malignant mammary tumours and relationship to clinicopathological features. The Veterinary Journal. 182 491-493.

Jurikovaa, M., Danihelb, L. and Polaka, S.2016. Ki67, PCNA, and MCM proteins: markers of proliferation in the diagnosis of breast cancer. Acta Histochemica.118:544-552.

Manuali, E., De Giuseppe, A, Feliziani, F, Forti, K, Casciari, C and Marchesi, M.C. CA 15-3 cell lines and tissue expression in canine mammary cancer and the correlation between serum levels and tumour histological grade. BMC Veterinary Research. 8:86.

Queiroga, F.L., Pires, I and Lobo, L. 2010. The role of Cox-2 expression in the prognosis of dogs with malignant mammary tumours. Research in Veterinary Science.88:441-445.

Rahn, J.J., Dabbagh, L, Pasdar, M, Hugh, J.C. 2001. The importance of MUC1 cellular localization in patients with breast carcinomaa: an immunohistologic study of 71 patients and review of the literature. Cancer. 1; 91(11):1973-82.

Rismanchi, S., Yadegar, O, Muhammadnejad, S, Amanpour, S, Taghizadeh-Jahed, M and Muhammadnejad, A. 2014. Expression of vimentin filaments in canine malignant mammary gland tumors: A simulation of clinicopathological features of human breast cancer. Biomedical reports. 2: 725-728.

Salas, Y., Marquez, A, Diaz, D and Romero, L. 2015. Epidemiological study of mammary tumors in female dogs diagnosed during the period 2002-2012. A growing animal health problem. PLoS ONE.

Satelli, A., and Li, S. 2011.Vimentin in cancer and its potential as a molecular target for cancer therapy. Cellular and Molecular Life Sciences. 68:3033-3046.

Shao, Y., Sun, X and He, Y. 2015. Elevated levels of serum tumor markers CEA and CA15-3 are prognostic parameters for different molecular subtypes of breast cancer. PLoS ONE. 15(27):531-535.

Sorenmo, K., 2003. Canine mammary gland tumors. The Veterinary clinics of North America. Small Animal Practice. 33:573-596.

Zuccari. D., Marcilia. V, Pavam, Ana Carolina BTerzian, Rodrigo. S, Pereira, Ruiz, C.M and Andrade JC. 2008. Immunohistochemical evaluation of ecadherin, Ki-67 and PCNA in canine mammary neoplasias: Correlation of prognostic factors and clinical outcome Pesq. Vet. Bras. 28(4):207-215.

\section{How to cite this article:}

Expression of Biomarkers in Canine Mammary Tumours. 2019. Expression of Biomarkers in Canine Mammary Tumours. Int.J.Curr.Microbiol.App.Sci. 8(05): 1464-1473.

doi: https://doi.org/10.20546/ijcmas.2019.805.168 